\title{
Multi-vehicle Control in a Strong Flowfield with Application to Hurricane Sampling
}

\author{
Levi DeVries* and Derek A. Paley ${ }^{\dagger}$ \\ University of Maryland, College Park, Maryland, 20742, U.S.A
}

\begin{abstract}
A major obstacle to path-planning and formation-control algorithms in multi-vehicle systems are strong flows in which the ambient flow speed is greater than the vehicle speed relative to the flow. This challenge is especially pertinent in the application of unmanned aircraft used for collecting targeted observations in a hurricane. The presence of such a flowfield may inhibit a vehicle from making forward progress relative to a ground-fixed frame, thus limiting the directions in which it can travel. Using a selfpropelled particle model in which each particle moves at constant speed relative to the flow, this paper presents results for motion coordination in a strong, known flowfield. We present the particle model with respect to inertial and rotating reference frames and provide for each case a set of conditions on the flowfield that ensure trajectory feasibility. Results from the Lyapunov-based design of decentralized control algorithms are presented for circular, folium, and spirograph trajectories, which are selected for their potential use as hurricane sampling trajectories. The theoretical results are illustrated using numerical simulations in an idealized hurricane model.
\end{abstract}

\section{Nomenclature}

$N \quad$ Number of particles in the system

$k \quad$ Particle index $k=1, \ldots, N$

$r_{k} \quad$ Position of $k^{\text {th }}$ particle with respect to inertial frame

$\tilde{r}_{k} \quad$ Position of $k^{\text {th }}$ particle with respect to rotating frame

\footnotetext{
${ }^{*}$ Graduate Student, Department of Aerospace Engineering, University of Maryland, and AIAA Student Member.

${ }^{\dagger}$ Assistant Professor, Department of Aerospace Engineering, University of Maryland, and AIAA Associate Fellow.
} 
$\dot{r}_{k} \quad$ Velocity of $k^{\text {th }}$ particle with respect to inertial frame

$\dot{\tilde{r}}_{k} \quad$ Velocity of $k^{\text {th }}$ particle with respect to rotating frame

$f_{k} \quad$ Flow velocity at $r_{k}$

$\tilde{f}_{k} \quad$ Flow velocity at $\tilde{r}_{k}$ relative to rotating frame

$\theta_{k} \quad$ Orientation of $k^{\text {th }}$ flow-relative velocity with respect to an inertial frame

$\tilde{\theta}_{k} \quad$ Orientation of $k^{\text {th }}$ flow-relative velocity with respect to a rotating frame

$\gamma_{k} \quad$ Orientation of $k^{\text {th }}$ total velocity with respect to an inertial frame

$\tilde{\gamma}_{k} \quad$ Orientation of $k^{\text {th }}$ total velocity with respect to a rotating frame

$u_{k} \quad$ Flow-relative steering control of $k^{\text {th }}$ particle with respect to an inertial frame

$\nu_{k} \quad$ Total steering control of $k^{\text {th }}$ particle with respect to an inertial frame

$\tilde{u}_{k} \quad$ Flow-relative steering control of $k^{\text {th }}$ particle with respect to a rotating frame

$\tilde{\nu}_{k} \quad$ Total steering control of $k^{\text {th }}$ particle with respect to a rotating frame

$s_{k} \quad$ Total speed of $k^{\text {th }}$ particle with respect to an inertial frame

$\tilde{h}_{k} \quad$ Total speed of $k^{\text {th }}$ particle with respect to a rotating frame

$\tilde{s}_{k} \quad$ Flow-relative speed of $k^{\text {th }}$ particle with respect to a rotating frame

$c_{k} \quad$ center of the $k^{\text {th }}$ particle's trajectory

$\Omega \quad$ Constant angular rate of rotating frame

$\kappa_{k} \quad$ Curvature of $k^{\text {th }}$ particle's trajectory

$\omega_{0} \quad$ Constant curvature of a circle of radius $\left|\omega_{0}\right|^{-1}$

$K \quad$ Control gain

$n \quad$ Number of lobes in a folium figure

a Radius of folium lobe

$i \quad$ Imaginary unit

$v_{\max }$ Maximum wind speed in idealized hurricane model

$r_{\max }$ Radius of maximum wind speed in idealized hurricane model

\section{Introduction}

Unmanned vehicles have shown great value in their ability to explore extreme environments that are too dangerous for manned vehicles. Autonomous underwater vehicles have shed light on some of the deepest trenches of the sea, ${ }^{25}$ while the Mars rovers continue to explore expanses of the martian landscape. Recently, autonomous vehicles have been proposed as a safe and effective way to penetrate extreme weather systems such as hurricanes and tornados. ${ }^{4}$ In 2006, an Aerosonde unmanned aircraft successfully penetrated a typhoon eyewall while streaming temperature, pressure, and windspeed data to a remote command center. ${ }^{16}$ In 2010, the Global Hawk UAV passed over Hurricane Earl. ${ }^{15}$ In addition smaller 
aerial vehicles are being utilized to sample within tornadic supercell thunderstorms. ${ }^{5}$

The assimilation of data from previously inaccessible regions of a storm presents the possibility of more accurate forecasts of both the intensity and trajectory of a weather system. For hurricanes, existing data sampling methods include the use of satellite imagery, manned flights, and more recently, remotely piloted unmanned flights. New data assimilation techniques are in development for remotely piloted Global Hawk flights, ${ }^{7}$ which loiter above the winds associated with a hurricane at altitudes of up to $65,000 \mathrm{ft} .{ }^{10}$ In manned flights, the aircraft eject one-time-use dropsondes to collect data at lower altitudes where it is too dangerous to fly. By deploying hundreds of dropsondes, meteorologists obtain vertical profiles of atmospheric data in the storm.

Autonomous unmanned aircraft have been proposed as a safer, more efficient alternative to manned flights for low-altitude penetration of hurricanes; ${ }^{16}$ collecting observations of temperature, humidity, and wind at altitudes lower than manned aircraft can safely fly. Replacing dropsondes with a smaller number of unmanned, reusable aircraft flying in a moving formation provides continuous, dynamic data from the hurricane. Unmanned platforms are designed to withstand the severe winds within the storm and may operate autonomously, using automatic control algorithms.

To sample a circulating flowfield for forecasting purposes requires azimuthal and radial sampling densities such that resulting measurements concisely characterize global properties. In the case of hurricane sampling, manned flights traverse figure-four patterns in order to sample each quadrant of the storm while minimizing the overall track length. ${ }^{26}$ To emulate this sampling strategy requires the development of control algorithms that drive sampling vehicles in non-convex formations. In addition, these trajectories must be feasible for the sampling platform in the presence of a strong wind.

In order to design steering algorithms for unmanned aircraft in a hurricane, we study a planar, self-propelled Newtonian particle model in a strong flowfield (i.e., a flow speed equal to or exceeding the vehicle speed relative to flow). We provide decentralized Lyapunov-based control algorithms that stabilize moving formations of multiple vehicles, extending previous work for moderate ${ }^{18}$ (flow speeds between $10 \%$ and $99 \%$ of vehicle speed) and flow-free models. ${ }^{1,2,21,27}$ In the flow-free model (flow speeds $<10 \%$ of vehicle speed), Sepulchre et al. ${ }^{21}$ provided theoretically justified algorithms for stabilization of synchronized, circular, and circular symmetric formations. Arranz et al. expanded this result to the family of radially contracting and expanding ${ }^{2}$ as well as spatially translating ${ }^{1}$ circular formations. Moreover, with the use of curvature functions, Paley et al. ${ }^{17}$ extended the multi-vehicle formation result to convex loops; Zhang ${ }^{27}$ used orbit functions to stabilize formations on smooth curves.

In the moderate flow regime, Frew et al. ${ }^{9}$ utilized Lyapunov analysis to generate guidance vector fields that globally stabilize circular loitering patterns. Paley and Peterson ${ }^{18}$ extended 
the stability of synchronized and circular formations to known, spatially-varying flowfields as well as circular symmetric formations in uniform flows. Peterson and Paley ${ }^{19}$ also extended synchronized and circular formation control to unknown and time-varying flowfields by implementing onboard flowfield estimators.

The results described below are applicable to more than environmental sampling of extreme weather systems. This work has applications in path planning for autonomous underwater gliders navigating strong currents ${ }^{6}$ and in navigation of fixed-wing aircraft in significant and unknown windfields. ${ }^{3,20}$ The presence of a strong flow inhibits a vehicle from making forward progress in some areas of the flowfield, thus limiting the range of directions in which it can travel. This presents an obstacle to formation control of multi-vehicle systems in that formations may not be feasible due to spatial or temporal variability in the flow.

The results of this paper rely on the use of Lyapunov-based control design, which utilizes scalar artificial potential functions whose value is minimized by the desired formation. ${ }^{13}$ We design state-feedback control laws that ensure negative semi-definiteness of the potential function's time derivative, invoking the invariance principle to establish the stability properties of the closed-loop system. ${ }^{13}$

The contributions of this paper are as follows: (1) we derive self-propelled particle dynamics in a strong flow with respect to inertial and rotating reference frames; (2) we provide kinematic conditions such that a particle can follow a desired trajectory in a strong, known flowfield; and (3) we present theoretically justified multi-vehicle control laws to stabilize hurricane sampling formations including circular, folium, and spirograph patterns. These results extends previous results for convex loops in a uniform moderate flow to closed curves with non-zero curvature in a spatially varying strong flow.

This paper is organized in the following manner. In Section II, we present a dynamic model of planar, self-propelled particle motion, first with respect to an inertial reference frame and then in a reference frame rotating at a constant angular rate. In Section III we derive the kinematic constraints a strong flowfield must satisfy such that a desired trajectory is feasible with respect to an inertial frame. In addition, we derive the kinematic constraints a trajectory must satisfy to be feasible with respect to a rotating reference frame. In Section IV we present Lyapunov-based decentralized multi-vehicle control algorithms for vehicles traveling in a strong flow. Section V assesses the feasibility of sampling trajectories in an idealized hurricane model and, when feasible, we simulate the particle motion under closedloop control. Section VI summarizes the paper and provides insight into ongoing work. 


\section{Self-Propelled Particle Dynamics in a Strong Flowfield}

In this section we review the inertial motion of a self-propelled particle subject to a strong external flowfield. We derive a model of self-propelled particle motion with respect to a reference frame rotating at a constant angular rate relative to an inertial frame. The rotating-frame dynamics form the foundation for analysis of prospective hurricane sampling patterns, discussed in Section V.

\section{A. Particle Motion With Respect to an Inertial Reference Frame}

This paper extends an existing dynamic model common to many works on collective motion. ${ }^{2,11,17,18,23}$ The model consists of a collection of $N$ self-propelled Newtonian particles. Each particle is subject to a control force normal to its velocity and travels at a constant speed relative to an external flowfield. We assume without loss of generality that the flow-relative speed is unity. Given a ground-fixed inertial reference frame $\mathcal{I}$ (which we identify with the complex plane), the $k^{\text {th }}$ particle's position is represented by the vector $r_{k}=x_{k}+i y_{k} \in \mathbb{C}$. We represent the $k^{\text {th }}$ particle's velocity by $\cos \left(\theta_{k}\right)+i \sin \left(\theta_{k}\right)=e^{i \theta_{k}}$, where $\theta_{k}$ is a point on the unit circle. The control force $u_{k}$ determines the particle's turn rate, i.e., $\dot{\theta}_{k}=u_{k}$. The equations of motion for the $k^{\text {th }}$ particle are ${ }^{\mathrm{a}}$

$$
\begin{aligned}
& \dot{r}_{k}=e^{i \theta_{k}} \\
& \dot{\theta}_{k}=u_{k}(\mathbf{r}, \boldsymbol{\theta}), \quad k=1, \ldots, N .
\end{aligned}
$$

In the presence of a known, time-invariant flowfield, $f_{k}=f\left(r_{k}\right) \in \mathbb{C}$, each particle's velocity is represented by the vector sum of its velocity relative to the flow and the flow velocity relative to the inertial frame $\mathcal{I}$, as illustrated in Figure 1 . The $k^{\text {th }}$ particle's motion is governed by the equations

$$
\begin{aligned}
& \dot{r}_{k}=e^{i \theta_{k}}+f_{k} \\
& \dot{\theta}_{k}=u_{k}(\mathbf{r}, \boldsymbol{\theta}), \quad k=1, \ldots, N .
\end{aligned}
$$

In this paper we allow $\left|f_{k}\right|$ to exceed one, which is the speed of the particle relative to the flow. Let $\gamma_{k}$ represents the orientation of the $k^{\text {th }}$ particle's inertial velocity and $s_{k}$ its magnitude. Previous work with moderate flows, ${ }^{18}$ i.e., where $\left|f_{k}\right|<1$, utilized the following coordinate transformation:

$$
\begin{aligned}
& \gamma_{k}=\arg \left(e^{i \theta_{k}}+f_{k}\right) \\
& s_{k}=\left|e^{i \theta_{k}}+f_{k}\right| .
\end{aligned}
$$

${ }^{\mathrm{a}}$ We use bold fonts to represent an $N \times 1$ matrix, e.g., $\boldsymbol{r}=\left[\begin{array}{llll}r_{1} & r_{2} & \ldots & r_{N}\end{array}\right]^{T}$ and $\boldsymbol{\theta}=\left[\begin{array}{llll}\theta_{1} & \theta_{2} & \ldots & \theta_{N}\end{array}\right]^{T}$. 


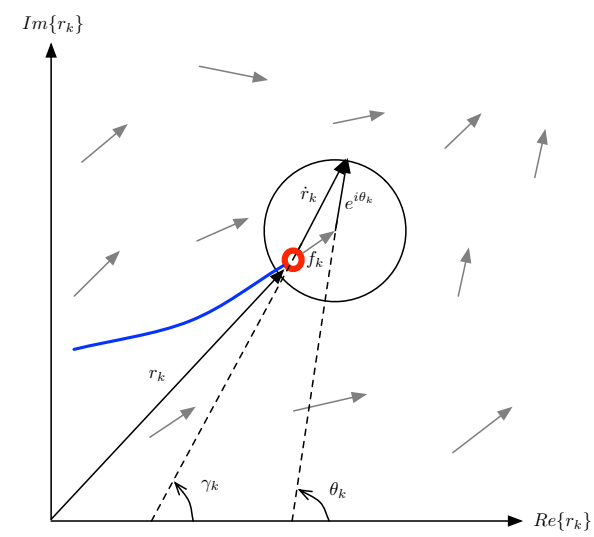

(a)

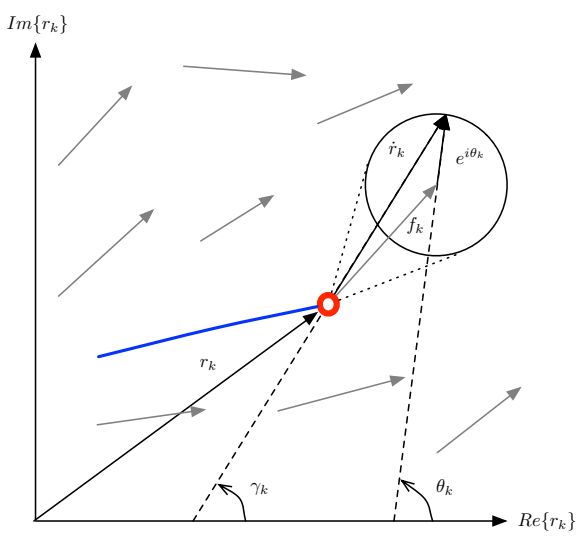

(b)

Figure 1. Illustration of particle motion in (a) moderate flow and (b) strong flow.

Under this transformation, the equations of motion are represented by

$$
\begin{aligned}
& \dot{r}_{k}=s_{k} e^{i \gamma_{k}} \\
& \dot{\gamma}_{k}=\nu_{k},
\end{aligned}
$$

where $\nu_{k}$ is the control relative to the fixed inertial frame $\mathcal{I}$. Controls $u_{k}$ and $\nu_{k}$ are related by $^{\mathrm{b}}$

$$
u_{k}=\frac{\nu_{k}-\left\langle f_{k}^{\prime}, i\right\rangle}{1-s_{k}^{-1}\left\langle e^{i \gamma_{k}}, f_{k}\right\rangle}=\frac{s_{k} \nu_{k}-s_{k}\left\langle f_{k}^{\prime}, i\right\rangle}{s_{k}-\left\langle e^{i \gamma_{k}}, f_{k}\right\rangle},
$$

where $f_{k}^{\prime}=\frac{\partial f_{k}}{\partial r_{k}}$. Figure 1 illustrates the particle model in both moderate and strong flow regimes. Note that a unit circle centered at the tip of $f_{k}$ represents the possible values of $\dot{r}_{k}=e^{i \theta_{k}}+f_{k}$.

Paley and Peterson ${ }^{18}$ showed that in a moderate flow the transformation (6) defines a one-to-one mapping between $u_{k}$ (the control relative to the flow) and $\nu_{k}$ (the control relative to the ground), since the denominator is never zero. This result allowed development of control laws for (2), extending those developed for the flow-free model (1). However, for flow speeds greater than or equal to the vehicle speed, the transformation in (5) is no longer one-to-one. To see this, consider a particle at position $r_{k}$ subject to a strong flow. The unit circle $\dot{r}_{k}=e^{i \theta_{k}}+f_{k}$ is not guaranteed to enclose $r_{k}$, which implies that the set of possible inertial velocity orientations is a subset of $\mathbb{S}^{1}$, similar to the cone of admissable directions presented by Bakolas and Tsiotras. ${ }^{3}$ That is, any given $\theta_{k}$ maps to a single $\gamma_{k}$, but any given $\gamma_{k}$ maps to one of two possible $\theta_{k}$ 's. The speed $s_{k}$ is required to determine $\theta_{k}$ as shown in Figure 1(b). We identify two singularities in the control transform (6) in Lemma 1.

\footnotetext{
${ }^{\mathrm{b}}\langle x, y\rangle \triangleq \operatorname{Re}\left(x^{*} y\right)$ where $x^{*}$ is the complex conjugate of $x$, denotes the inner product of complex numbers $x$ and $y$.
} 
Lemma 1. For $\left|f_{k}\right| \geq 1$, the control transform (6) is singular when $\theta_{k}=\arg \left(f_{k}\right) \pm$ $\cos ^{-1}\left(\left|f_{k}\right|^{-1}\right)$.

Proof. See Appendix.

Lemma 1 shows that strong flows introduce singularities in the coordinate transform (6), resulting in an unbounded turn rate. However, the design of the vehicle and the medium in which it travels dictate the maximum turn rate a vehicle can have. This constraint can be modeled as a saturation function on $u_{k}$ such that (2) becomes

$$
\begin{aligned}
& \dot{r}_{k}=e^{i \theta_{k}}+f_{k} \\
& \dot{\theta}_{k}=\operatorname{sat}\left\{u_{k}(\mathbf{r}, \boldsymbol{\theta}) ; u_{\max }\right\}
\end{aligned}
$$

where

$$
\operatorname{sat}\left\{u ; u_{\max }\right\}= \begin{cases}u_{\max }, & u>u_{\max } \\ u, & -u_{\max } \leq u \leq u_{\max } \\ -u_{\max }, & u<-u_{\max } .\end{cases}
$$

The model (7) allows us to use (5) to design $\nu_{k}$ and (6) to map $\nu_{k}$ to $u_{k}$. $u_{\max }$ can be chosen arbitrarily large, and must be chosen large enough so that $\nu_{\max }=\nu_{\max }\left(u_{\max }\right)>$ $\left|\kappa_{\max } s_{\max }\right|$, where $\kappa_{\max }$ is the maximum desired curvature of the particle trajectory and $s_{\max }$ the maximum particle speed along the trajectory. (The relation between $u_{\max }$ and $\nu_{\max }$ is discussed by Peterson and Paley. ${ }^{19}$ ) We represent the upper bound on $\nu_{k}$ using $\dot{\gamma}_{k}=\operatorname{sat}\left\{\nu(\mathbf{r}, \theta) ; \nu_{\max }\right\}$. Using $(7)$, as $\theta_{k}$ approaches a singularity, $\dot{\theta}_{k}$ remains bounded, passing through the singular point at a constant rate $\pm u_{\max }$.

\section{B. Particle Motion with Respect to a Rotating Reference Frame}

To achieve adequate radial and azimuthal sampling within a circulating flowfield, a proposed sampling formation is that of a spirograph. When viewed with respect to a frame rotating at constant angular rate, a spirograph trajectory is simply a circular trajectory. The control algorithms producing circular trajectories and formations in a non-rotating frame are well studied, ${ }^{21}$ and reviewed in Section IV. Here, we develop a dynamic model of self-propelled particles in a rotating reference frame. The control variable $\tilde{u}_{k}$ regulates the turn rate of the $k^{\text {th }}$ particle. We return to the design of a spirograph control in Section IV.

Let $\mathcal{I}=\left(O, \mathbf{e}_{1}, \mathbf{e}_{2}, \mathbf{e}_{3}\right)$ represent an inertial reference frame with origin $O$ and $\mathcal{B}=$ $\left(O, \mathbf{b}_{1}, \mathbf{b}_{2}, \mathbf{b}_{3}\right)$, where $\mathbf{b}_{3}=\mathbf{e}_{3}$, represent a rotating reference frame with angular velocity ${ }^{\mathcal{I}} \boldsymbol{\omega}^{\mathcal{B}}=\Omega \mathbf{e}_{3}$. (When convenient we use complex notation to represent cartesian coordinates in $\mathcal{I}$ and $\mathcal{B}$.) The orientation $\alpha=\Omega t+\alpha(0)$ of frame $\mathcal{B}$ with respect to $\mathcal{I}$ satisfies $\mathbf{e}_{1} \cdot \mathbf{b}_{1}=\cos \alpha$, as shown in Figure 2(a). The inertial kinematics of the $k^{\text {th }}$ particle with respect to $O$ are 


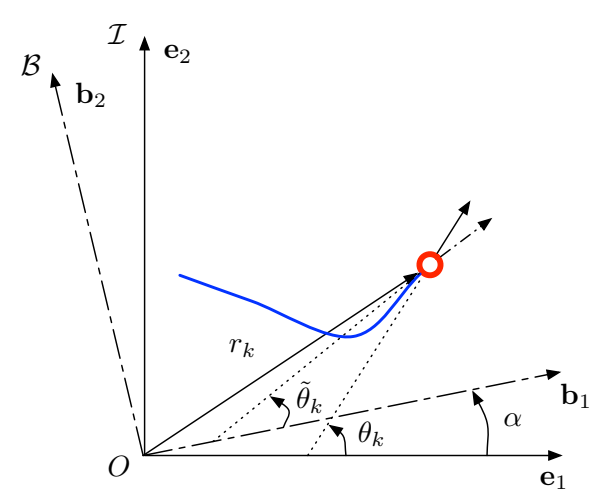

(a)

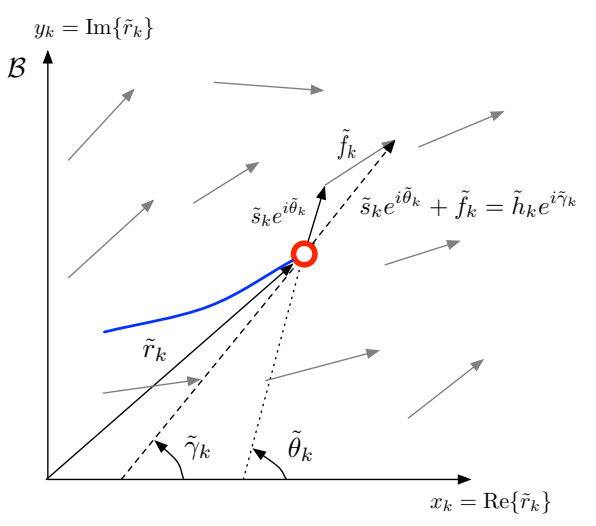

(b)

Figure 2. The orientation of the $k^{\text {th }}$ particle's velocity (a) in no flow and (b) in flow $\tilde{f}$.

described by $\mathbf{r}_{k / O}$ and ${ }^{{ }^{\mathcal{I}}} \mathbf{v}_{k / O}=\frac{{ }^{I} d}{d t}\left(\mathbf{r}_{k / O}\right)$, where $r_{k}=\left[\mathbf{r}_{k / O}\right]_{\mathcal{I}}$ is the position expressed in (complex) coordinates with respect to frame $\mathcal{I}$. We define a path frame $C=\left(k, \mathbf{c}_{1}, \mathbf{c}_{2}, \mathbf{c}_{3}\right)$, where $\mathbf{c}_{3}=\mathbf{e}_{3}, \mathbf{c}_{1}={ }^{\mathcal{I}} \mathbf{v}_{k / O} / s_{k}$ and $s_{k}=\left\|^{\mathcal{I}} \mathbf{v}_{k / O}\right\|$ is the speed of the particle, equal to one when no flow is present. Let $\theta_{k}$ be the orientation of the inertial velocity so that $\mathbf{e}_{1} \cdot \mathbf{c}_{1}=\cos \theta_{k}$.

Since frame $\mathcal{B}$ shares its origin with $\mathcal{I}$, the kinematics of $k$ in frame $\mathcal{B}$ are given by $\mathbf{r}_{k / O}$ and ${ }^{\mathcal{B}} \mathbf{v}_{k / O}=\frac{{ }^{\mathcal{B}} d}{d t}\left(\mathbf{r}_{k / O}\right)$. Similarly, this gives rise to the path frame $D=\left(k, \mathbf{d}_{1}, \mathbf{d}_{2}, \mathbf{d}_{3}\right)$, where $\mathbf{d}_{3}=\mathbf{e}_{3}, \mathbf{d}_{1}={ }^{\mathcal{B}} \mathbf{v}_{k / O} / \tilde{s}_{k}$ and $\tilde{s}_{k}=\left\|^{\mathcal{B}} \mathbf{v}_{k / O}\right\|$ represents the speed of the particle relative to frame $\mathcal{B}$. In $\mathcal{B}$-frame coordinates, $\left(x_{k}, y_{k}\right)_{\mathcal{B}}$, we have $\mathbf{r}_{k / O}=x_{k} \mathbf{b}_{1}+y_{k} \mathbf{b}_{2}$ and ${ }^{\mathcal{B}} \mathbf{v}_{k / O}=\dot{x}_{k} \mathbf{b}_{1}+\dot{y}_{k} \mathbf{b}_{2}$, which implies $\tilde{s}_{k}=\sqrt{\dot{x}_{k}^{2}+\dot{y}_{k}^{2}}$. Let $\tilde{\theta}_{k}$ be the orientation of ${ }^{\mathcal{B}} \mathbf{v}_{k / O}$ relative to $\mathbf{b}_{1}$, so that $\mathbf{b}_{1} \cdot \mathbf{d}_{1}=\cos \tilde{\theta}_{k}$.

The transport equation ${ }^{12}{ }^{\mathcal{I}} \mathbf{v}_{k / O}={ }^{\mathcal{B}} \mathbf{v}_{k / O}+{ }^{\mathcal{I}} \boldsymbol{\omega}^{\mathcal{B}} \times \mathbf{r}_{k / O}$ gives the kinematic relationship

$$
s_{k} \mathbf{c}_{1}=\tilde{s}_{k} \mathbf{d}_{1}+\Omega \mathbf{b}_{3} \times\left(x_{k} \mathbf{b}_{1}+y_{k} \mathbf{b}_{2}\right)=\tilde{s}_{k} \mathbf{d}_{1}+\Omega\left(x_{k} \mathbf{b}_{2}-y_{k} \mathbf{b}_{1}\right)
$$

Noting that $\mathbf{c}_{1}=\cos \theta_{k} \mathbf{e}_{1}+\sin \theta_{k} \mathbf{e}_{2}$, (9) can be written

$$
s_{k} \cos \left(\theta_{k}-\alpha_{k}\right) \mathbf{b}_{1}+s_{k} \sin \left(\theta_{k}-\alpha_{k}\right) \mathbf{b}_{2}=\left(\tilde{s}_{k} \cos \tilde{\theta}_{k}-\Omega y_{k}\right) \mathbf{b}_{1}+\left(\tilde{s}_{k} \sin \tilde{\theta}_{k}+\Omega x_{k}\right) \mathbf{b}_{2}
$$

The inertial derivative of (9), assuming $s_{k}$ is constant, gives

$$
s_{k} \dot{\theta}_{k} \mathbf{c}_{2}=\dot{\tilde{s}}_{k} \mathbf{d}_{1}+\tilde{s}_{k}\left(\dot{\tilde{\theta}}_{k}+\Omega\right) \mathbf{d}_{2}-\left(\Omega \dot{y}_{k}+\Omega^{2} x_{k}\right) \mathbf{b}_{1}+\left(\Omega \dot{x}_{k}-\Omega^{2} y_{k}\right) \mathbf{b}_{2},
$$

which by utilizing $\mathbf{c}_{2}=-\sin \left(\theta_{k}-\alpha\right) \mathbf{b}_{1}+\cos \left(\theta_{k}-\alpha\right) \mathbf{b}_{2}$ results in the scalar equations of 
motion relative to frame $\mathcal{B}$ :

$$
\begin{aligned}
-s_{k} u_{k} \sin (\theta-\alpha) & =\dot{\tilde{s}}_{k} \cos \tilde{\theta}_{k}-\tilde{s}_{k}\left(\dot{\tilde{\theta}}_{k}+\Omega\right) \sin \tilde{\theta}_{k}-x_{k} \Omega^{2}-\dot{y}_{k} \Omega \\
s_{k} u_{k} \cos \left(\theta_{k}-\alpha\right) & =\dot{\tilde{s}}_{k} \sin \tilde{\theta}_{k}+\tilde{s}_{k}\left(\dot{\tilde{\theta}}_{k}+\Omega\right) \cos \tilde{\theta}_{k}-y_{k} \Omega^{2}+\dot{x}_{k} \Omega
\end{aligned}
$$

Note that substituting $\dot{x}_{k}=\tilde{s}_{k} \cos \tilde{\theta}_{k}$ and $\dot{y}_{k}=\tilde{s}_{k} \sin \tilde{\theta}_{k}$ into (10) to eliminate $\theta_{k}-\alpha$ and $s_{k}$ gives

$$
\begin{aligned}
-u_{k} \tilde{s}_{k} \sin \tilde{\theta}_{k}-u_{k} \Omega x_{k} & =\dot{\tilde{s}}_{k} \cos \tilde{\theta}_{k}-\tilde{s}_{k}\left(\dot{\tilde{\theta}}_{k}+\Omega\right) \sin \tilde{\theta}_{k}-x_{k} \Omega^{2}-\tilde{s}_{k} \sin \tilde{\theta}_{k} \Omega \\
u_{k} \tilde{s}_{k} \cos \tilde{\theta}_{k}-u_{k} \Omega y_{k} & =\dot{\tilde{s}}_{k} \sin \tilde{\theta}_{k}+\tilde{s}_{k}\left(\tilde{\tilde{\theta}}_{k}+\Omega\right) \cos \tilde{\theta}_{k}-y_{k} \Omega^{2}+\tilde{s}_{k} \cos \tilde{\theta}_{k} \Omega
\end{aligned}
$$

Solving (12) for $\dot{\tilde{s}}_{k}$ and $\dot{\tilde{\theta}}_{k}$, respectively, with complex notation $\tilde{r}_{k}=x_{k}+i y_{k}=\left[\mathbf{r}_{k / O}\right]_{\mathcal{B}}$ results in the equations of motion of the $k^{\text {th }}$ particle relative to frame $\mathcal{B}$ :

$$
\begin{aligned}
& \dot{\tilde{r}}_{k}=\tilde{s}_{k} e^{i \tilde{\theta}_{k}} \\
& \dot{\tilde{s}}_{k}=\left(\Omega^{2}-u_{k} \Omega\right)\left\langle\tilde{r}_{k}, e^{i \tilde{\theta}_{k}}\right\rangle \\
& \dot{\tilde{\theta}}_{k}=u_{k}-2 \Omega+\tilde{s}_{k}^{-1}\left(\Omega^{2}-u \Omega\right)\left\langle\tilde{r}_{k}, i e^{i \tilde{\theta}_{k}}\right\rangle \triangleq \tilde{u}_{k} .
\end{aligned}
$$

We define the flow-relative control variable with respect to the rotating frame, $\tilde{u}_{k}$, such that the mapping to the inertial control $u_{k}$ is

$$
u_{k}=\frac{\tilde{u}_{k} \tilde{s}_{k}+2 \Omega \tilde{s}_{k}-\Omega^{2}\left\langle\tilde{r}_{k}, i e^{i \tilde{\theta}_{k}}\right\rangle}{\tilde{s}_{k}-\Omega\left\langle\tilde{r}_{k}, i e^{i \tilde{\theta}_{k}}\right\rangle} .
$$

Equations (13) and (14) are the equations of motion of an inertially unit-speed vehicle represented in coordinates relative to frame $\mathcal{B}$ rotating with constant angular rate $\Omega$.

Note that the mapping from $\tilde{u}_{k}$ to $u_{k}$ is singular when $\tilde{s}_{k}=\Omega\left\langle\tilde{r}_{k}, i e^{i \tilde{\theta}_{k}}\right\rangle$. The following result establishes the singular conditions with respect to the inertial speed $s_{k}$.

Lemma 2. The control transform (14) is singular when the speed of the particle in a flow-free inertial frame is $s_{k}=\Omega\left\langle\tilde{r}_{k}, e^{i \tilde{\theta}_{k}}\right\rangle$.

Proof. See Appendix.

The singularity identified in Lemma 2 occurs when the speed of the vehicle is zero with respect to frame $\mathcal{B}$. For example, when $e^{i \tilde{\theta}_{k}}$ is normal to $\tilde{r}_{k}$ the singular condition simplifies to $\Omega^{-1}=\left|\tilde{r}_{k}\right|$, which for a unit speed particle is equivalent to the familiar equation of the velocity of a rotating particle constrained to a circle.

In the presence of an external flowfield, the dynamics presented in (13) must also include flow terms. With respect to the rotating frame, the inertial flowfield is represented via the 
transport equation $^{12}$ such that

$$
f_{k}=\tilde{f}_{k}+{ }^{\mathcal{I}} \boldsymbol{\omega}^{\mathcal{B}} \times \tilde{r}_{k}
$$

where $\tilde{f}_{k}$ represents the flowfield relative to the rotating frame. Similar to (2) the flowfield is incorporated into (13) such that we achieve

$$
\begin{aligned}
& \dot{\tilde{r}}_{k}=\tilde{s}_{k} e^{i \tilde{\theta}_{k}}+\tilde{f}_{k} \\
& \dot{\tilde{s}}_{k}=\left(\Omega^{2}-u_{k} \Omega\right)\left\langle\tilde{r}_{k}, e^{i \tilde{\theta}_{k}}\right\rangle \\
& \dot{\tilde{\theta}}_{k}=\tilde{u}_{k},
\end{aligned}
$$

where $u_{k}$ is calculated from $\tilde{u}_{k}$ using (14).

We desire to control the direction of the total velocity of the particle rather than the flow-relative velocity. For this reason let

$$
\begin{aligned}
& \tilde{h}_{k} \triangleq\left|\tilde{s}_{k} e^{i \tilde{\theta}_{k}}+\tilde{f}_{k}\right| \\
& \tilde{\gamma}_{k} \triangleq \arg \left(\tilde{s}_{k} e^{i \tilde{\theta}_{k}}+\tilde{f}_{k}\right),
\end{aligned}
$$

such that $\dot{\tilde{r}}_{k}=\tilde{h}_{k} e^{i \tilde{\gamma}_{k}}$. Figure 2(b) illustrates the following relations

$$
\begin{aligned}
\tilde{h}_{k} \cos \tilde{\gamma}_{k} & =\tilde{s}_{k} \cos \tilde{\theta}_{k}+\left\langle\tilde{f}_{k}, 1\right\rangle \\
\tilde{h}_{k} \sin \tilde{\gamma}_{k} & =\tilde{s}_{k} \sin \tilde{\theta}_{k}+\left\langle\tilde{f}_{k}, i\right\rangle,
\end{aligned}
$$

which after division yield

$$
\tan \tilde{\gamma}_{k}=\frac{\tilde{s}_{k} \sin \tilde{\theta}_{k}+\left\langle\tilde{f}_{k}, i\right\rangle}{\tilde{s}_{k} \cos \tilde{\theta}_{k}+\left\langle\tilde{f}_{k}, 1\right\rangle} .
$$

Taking the time derivative of (18) and using (17) gives

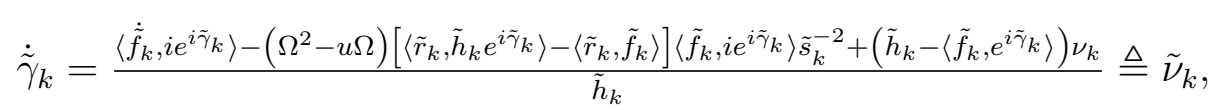

where $\tilde{\nu}_{k}$ represents control of the total velocity orientation with respect to the rotating frame and $\dot{\tilde{f}}_{k}=\frac{\partial \tilde{f}_{k}}{\partial \tilde{r}_{k}} \dot{\tilde{r}}_{k}$. Using (19) with (14) yields the following mapping between $u_{k}$ and $\tilde{\nu}_{k}$ necessary for implementing a control designed in the rotating frame for use in the inertial frame:

$$
u_{k}=\frac{\tilde{h}_{k} \tilde{\nu}_{k}-\left\langle\dot{\tilde{f}}_{k}, i e^{i \gamma_{k}}\right\rangle+2 \Omega\left(\tilde{h}_{k}-\left\langle\tilde{f}_{k}, i e^{i \gamma_{k}}\right\rangle\right)+\Omega^{2}\left\langle\tilde{r}_{k}, \tilde{h}_{k} e^{i \gamma_{k}}-\tilde{f}_{k}\right\rangle\left\langle\tilde{f}_{k}, i e^{i \tilde{\gamma}_{k}}\right\rangle \tilde{s}_{k}^{-2}-\Omega^{2}\left(\tilde{h}_{k}-\left\langle\tilde{f}_{k}, e^{i \tilde{\gamma}_{k}}\right\rangle\right)\left\langle\tilde{r}_{k}, i e^{i \tilde{\theta}_{k}}\right\rangle \tilde{s}_{k}^{-1}}{\tilde{h}_{k}-\left\langle\tilde{f}_{k}, e^{i \tilde{\gamma}_{k}}\right\rangle-\Omega\left|\tilde{f}_{k}\right|^{2} \tilde{s}_{k}^{-2}\left\langle\tilde{r}_{k}, i e^{i \tilde{\gamma}_{k}}\right\rangle+\Omega \tilde{h}_{k} \tilde{s}_{k}^{-2}\left(\left\langle\tilde{r}_{k}, i \tilde{f}_{k}\right\rangle+\left\langle\tilde{r}_{k}, e^{i \tilde{\gamma}_{k}}\right\rangle\left\langle\tilde{f}_{k}, i e^{i \tilde{\gamma}_{k}}\right\rangle+\left\langle\tilde{f}_{k}, e^{i \tilde{\gamma}_{k}}\right\rangle\left\langle\tilde{r}_{k}, i e^{i \tilde{\gamma}_{k}}\right\rangle\right)-\Omega \tilde{h}_{k} \tilde{s}_{k}^{-2}\left\langle\tilde{r}_{k}, i e^{\left.i \tilde{\gamma}_{k}\right\rangle}\right.}
$$

We now write the equations of motion of particle $k$ with respect to a rotating frame 


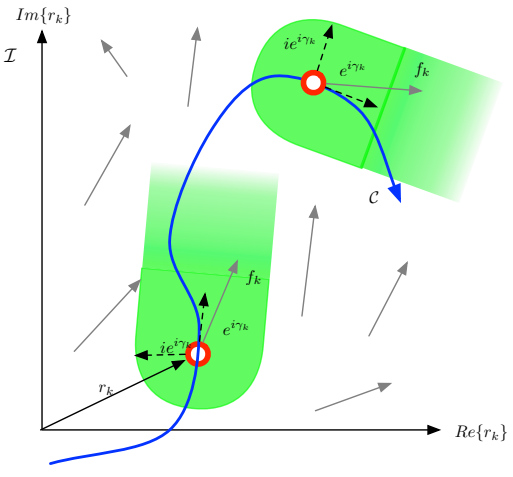

(a)

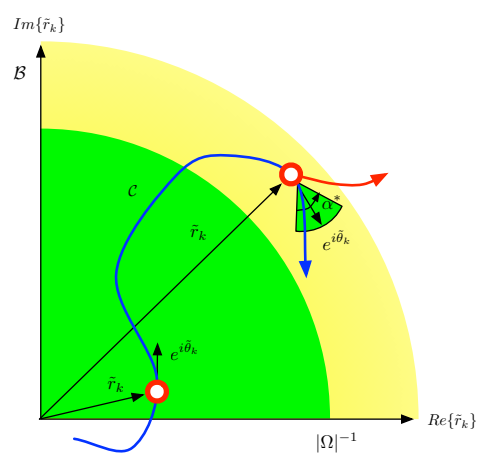

(b)

Figure 3. Feasibility constraint for (a) inertial frame and (b) rotating flow-free frame.

subject to a flowfield:

$$
\begin{aligned}
& \dot{\tilde{r}}_{k}=\tilde{h}_{k} e^{i \tilde{\gamma}_{k}} \\
& \dot{\tilde{s}}_{k}=\left(\Omega^{2}-u_{k} \Omega\right)\left\langle\tilde{r}_{k}, \tilde{h}_{k} e^{i \tilde{\gamma}_{k}}-\tilde{f}_{k}\right\rangle \tilde{s}_{k}^{-1} \\
& \dot{\tilde{\gamma}}_{k}=\tilde{\nu}_{k},
\end{aligned}
$$

where $u_{k}$ is given by (20). Similar to the inertial-frame dynamics, saturation is used to avoid singularities between control mappings (14) and (20).

For a known, spatially varying flowfield $\tilde{h}_{k}$ can be calculated as follows. ${ }^{18}$ Let $\tilde{f}_{k}=\alpha_{k}+i \beta_{k}$ represent the flowfield at position $\tilde{r}_{k}$ where $\alpha_{k}=\left\langle\tilde{f}_{k}, 1\right\rangle$ and $\beta_{k}=\left\langle\tilde{f}_{k}, i\right\rangle$ represent the real and imaginary parts of the flow respectively. By definition,

$$
\tilde{h}_{k}=\sqrt{\tilde{s}_{k}^{2}-\alpha_{k}^{2}-\beta_{k}^{2}+2 \tilde{h}_{k}\left(\alpha_{k} \cos \tilde{\gamma}_{k}+\beta_{k} \sin \tilde{\gamma}_{k}\right)} .
$$

Squaring this result and utilizing the quadratic formula to solve for $\tilde{h}_{k}$ gives

$$
\tilde{h}_{k}=\left\langle e^{i \tilde{\gamma}_{k}}, \tilde{f}_{k}\right\rangle+\sqrt{\tilde{s}_{k}^{2}-\left\langle i e^{i \tilde{\gamma}_{k}}, \tilde{f}_{k}\right\rangle^{2}}
$$

where we take the positive root since $\tilde{h}_{k}>0$.

\section{Trajectory Feasibility in a Strong Flow}

Strong flows present the possibility that a desired trajectory or formation is not feasible. In this section, we derive the kinematic conditions a flowfield must satisfy to ensure trajectory feasibility. Conditions are derived with respect to both inertial and rotating reference frames. These results have similarities to that of Bakolas and Tsiotras in assessing the reachability of 
two points within a flowfield for a kinematic model of an aircraft, ${ }^{3}$ but we consider flowfields that can vary continuously through space rather than flowfields that are assumed to be regionally uniform.

\section{A. Feasibility With Respect to an Inertial Reference Frame}

As a motivating example, in a strong uniform flow, a vehicle has no recourse but to be swept downwind. This presents a challenge to coordinated motion in that individual vehicles may not be able to reach the desired formation. Moreover, the desired trajectory itself may not be achievable if even a portion of the trajectory opposes the flow. The following analysis describes a set of constraints the flow must satisfy such that a given desired trajectory is feasible.

For a unit-speed vehicle to travel along $\mathcal{C}$, the flow at every point on the path must be such that the vehicle can maintain forward progress with velocity tangent to $\mathcal{C}$. That is, for every point on the desired trajectory, the component of the flow normal to $\mathcal{C}$ must be less than or equal to one. Mathematically, this implies that the absolute value of the inner product of the normal vector $i e^{i \gamma_{k}}$ and the flow must satisfy $\left|\left\langle i e^{i \gamma_{k}}, f\right\rangle\right| \leq 1$. If $\left\langle e^{i \gamma_{k}}, f_{k}\right\rangle<0$, then the flow opposes the direction of the trajectory and must therefore have magnitude less than one for the vehicle to make forward progress along $\mathcal{C}$. If $\left\langle e^{i \gamma_{k}}, f_{k}\right\rangle \geq 0$, then the flow must only satisfy the normal constraint. This result is summarized as follows:

Theorem 1. Trajectory $\mathcal{C}$ is feasible in flowfield $f$ if for all $r_{k} \in \mathcal{C}, f_{k}=f\left(r_{k}\right)$ satisfies

$$
\begin{aligned}
\left\langle e^{i \gamma_{k}}, f_{k}\right\rangle & <0 \text { and }\left|f_{k}\right|<1, \text { or } \\
& \geq 0 \text { and }\left|\left\langle i e^{i \gamma_{k}}, f_{k}\right\rangle\right| \leq 1
\end{aligned}
$$

where $e^{i \gamma_{k}}$ is tangent to $\mathcal{C}$ at $r_{k}$.

Theorem 1 implies that the flow vector at a given point on the trajectory must lie within a U-shaped envelope oriented along the trajectory, shown by the green-shaded region in Figure 3(a). Trajectories that do not satisfy (24) are not feasible. In a known, time-invariant flowfield Theorem 1 allows one to quantify regions of the flowfield in which parametric families of feasible trajectories are found.

To illustrate Theorem 1 we evaluate (24) for a family of circular trajectories in a strong flowfield. Analysis over the entire space of candidate circle centers produces a map of regions in which feasible trajectories can be achieved. This analysis is shown in Figure 4 for an arbitrary, strong flowfield. Circles whose center lie in a shaded region are not feasible; unshaded regions are feasible. Three sample trajectories are plotted. Red arrows indicate where the flow fails the constraints given in Theorem 1 . 


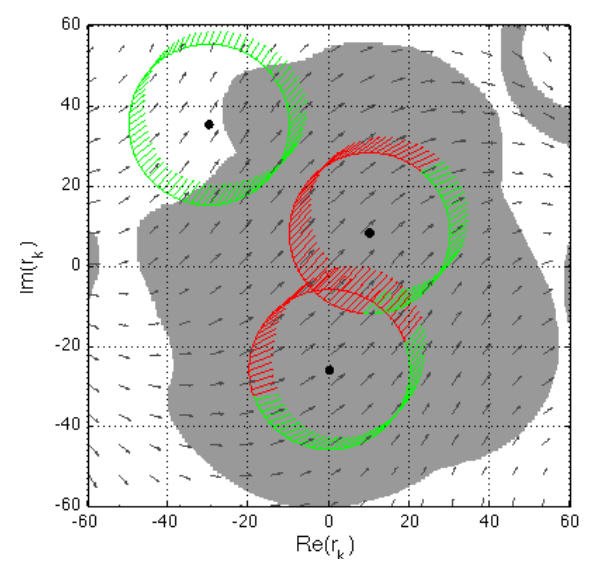

(a)

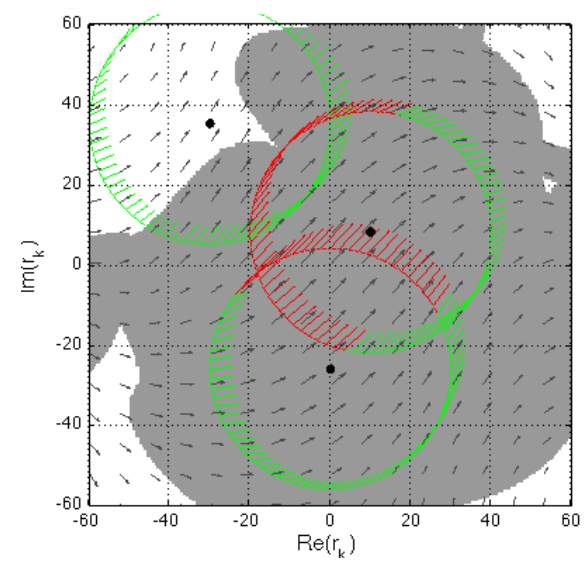

(b)

Figure 4. Feasible regions for counterclockwise circular trajectories (a) radius $\left|\omega_{0}\right|^{-1}=20$ and (b) $\left|\omega_{0}\right|^{-1}=30$.

\section{B. Feasibility with Respect to a Rotating Reference Frame}

In Section II we derive the dynamics of a self propelled particle with respect to a rotating reference frame. The equations of motion in (13) reveal that the speed of the particle in the rotating frame is a dynamic variable whose time derivative depends on the position and velocity of the particle. In this subsection we define the kinematic constraints on motion with respect to a rotating reference frame in a flow-free setting.

In the absence of an external flow, the kinematic terms arising from the rotating reference frame affect the speed $\tilde{s}_{k}$ of the vehicle with respect to the rotating frame. For a constantspeed particle traveling in the direction of rotation, the speed of the particle with respect to a rotating reference frame is a decreasing function of the rotation rate and the distance to the center of rotation. For a given trajectory to be feasible with respect to the rotating frame, a particle must maintain forward progress over the entire trajectory, which implies the following result.

Lemma 3. Trajectory $\mathcal{C}$ is feasible with respect to (13) if, for all $\tilde{r}_{k} \in\left\{\left.\tilde{r}_{k} \in \mathcal{C}|| \tilde{r}_{k}|\geq| \Omega\right|^{-1}\right\}$,

$$
\Omega\left\langle\tilde{r}_{k}, i e^{i \tilde{\theta}_{k}}\right\rangle>0 \text { and }\left|\Omega\left\langle\tilde{r}_{k}, e^{i \tilde{\theta}_{k}}\right\rangle\right| \leq 1
$$

where $e^{i \tilde{\theta}_{k}}$ is tangent to $\mathcal{C}$ at $\tilde{r}_{k}$.

Proof. Summing the squared components of (10) and using $s_{k}=1$ yields

$$
1=\tilde{s}_{k}^{2}+\Omega^{2}\left(x^{2}+y^{2}\right)+2 \Omega \tilde{s}_{k}
$$


Completing the square to solve for $\tilde{s}_{k}$ and simplifying with $\tilde{r}_{k}=x_{k}+i y_{k}$ gives

$$
\tilde{s}_{k}=\Omega\left\langle\tilde{r}_{k}, i e^{i \tilde{\theta}_{k}}\right\rangle+\sqrt{1-\Omega^{2}\left\langle\tilde{r}_{k}, e^{i \tilde{\theta}_{k}}\right\rangle^{2}}
$$

where the positive root is taken since $\tilde{s}_{k}>0$ is required to maintain forward progress. Notice that the inner products $\left\langle\tilde{r}_{k}, i e^{i \tilde{\theta}_{k}}\right\rangle$ and $\left\langle\tilde{r}_{k}, e^{i \tilde{\theta}_{k}}\right\rangle$ differ in phase by $\pi / 2$ which implies that for $\left|\tilde{r}_{k}\right|<|\Omega|^{-1}$, (26) is strictly greater than zero. A trajectory is infeasible if $\operatorname{Im}\left(\tilde{s}_{k}\right) \neq 0$ or $\tilde{s}_{k}<0$, implying that for $\left|\tilde{r}_{k}\right| \geq|\Omega|^{-1}$ a feasible trajectory must satisfy (25).

Lemma 3 is illustrated for $\Omega>0$ in Figure 3(b). Feasible trajectories with $\left|\tilde{r}_{k}\right| \geq|\Omega|^{-1}$ must travel in a direction opposing the rate of rotation in order to maintain forward progress with respect to the rotating frame. An infeasible path is shown in red. The range of feasible directions of travel is determined by the position of the vehicle and rate of rotation of the rotating frame. Notice that in complex notation (10) can be written $e^{i\left(\theta_{k}-\alpha\right)}=$ $\tilde{s}_{k} e^{i \tilde{\theta}_{k}}+i \Omega \tilde{r}_{k}$. Therefore, we can represent the velocity with respect to the rotating frame as $\tilde{s}_{k} e^{i \tilde{\theta}_{k}}=e^{i\left(\theta_{k}-\alpha\right)}-i \Omega \tilde{r}_{k}$. Thus, the range of feasible velocities with respect to the rotating frame is defined by a unit circle drawn about the tip of $-i \Omega \tilde{r}_{k}$. It can be shown that for $\left|\tilde{r}_{k}\right|>|\Omega|^{-1}$ the range of feasible directions of travel $\alpha^{*}$ about $\arg \left(-i \Omega \tilde{r}_{k}\right)$ is given by $\alpha^{*}=2 \sin ^{-1}\left(\left|\Omega \tilde{r}_{k}\right|^{-1}\right)$.

\section{Multi-vehicle Control in a Strong Flowfield}

In this section we review the design of multi-vehicle control for a moderate flowfield and derive circular and folium control laws for the particle model (2) in a strong flowfield. The results of this section are also applicable to the model (16) for a rotating reference frame as discussed in Section V.

\section{A. Review of Curvature Control in a Moderate Flowfield}

In this section we describe individual particle curvature control in a moderate flow, following the work of Paley et al., ${ }^{17}$ who produced decentralized algorithms to stabilize the family of convex loops called superellipses in a flow-free environment and Techy et al., ${ }^{23}$ where the convex-loop results were first extended to the vehicle model with flow (5).

Our goal is to drive the $k^{\text {th }}$ particle about a smooth, closed curve $\mathcal{C}$ with definite, bounded curvature, $\kappa_{k}$. To do so, $\mathcal{C}$ is parameterized by its center $c_{k}$. In a $c_{k}$-centered reference frame the particle position $\rho_{k}$ is parameterized by the central or polar angle, $\phi_{k}$. For a closed curve $\mathcal{C}$, completion of one rotation about sweeps through $2 \pi$ radians. Thus, $\rho_{k}(\phi) \in \mathbb{C}$ where $\phi_{k} \in[0,2 \pi)$. The orientation of the particle's inertial velocity relative to the $c_{k}$ centered

frame is $\gamma_{k}$ and we assume a smooth mapping $\gamma_{k} \mapsto \phi\left(\gamma_{k}\right)$ exists. The derivative $\frac{d \rho_{k}}{\mathrm{~d} \phi_{k}}$ is 
tangent to $\mathcal{C}$, implying the constraint $e^{i \gamma_{k}}=\left|\frac{d \rho_{k}}{d \phi_{k}}\right|^{-1} \frac{d \rho_{k}}{d \phi_{k}}$.

The local curvature is

$$
\kappa_{k}\left(\phi_{k}\right)= \pm \frac{d \gamma_{k}}{d \sigma}
$$

where

$$
\sigma\left(\phi_{k}\right)=\int_{0}^{\phi_{k}}\left|\frac{d \rho}{d \bar{\phi}}\right| d \bar{\phi}
$$

is the arc length along the curve. Note that the sign of $\kappa_{k}$ determines the direction of rotation about the curve clockwise or counterclockwise. Equations (27) and (28) imply

$$
\kappa_{k}^{-1}= \pm \frac{d \sigma_{k}}{d \phi_{k}} \frac{d \phi_{k}}{d \gamma_{k}}= \pm\left|\frac{d \rho_{k}}{d \phi_{k}}\right| \frac{d \phi_{k}}{d \gamma_{k}} .
$$

Under the tangent constraint, (29) can be written

$$
e^{i \gamma_{k}} \kappa_{k}^{-1}= \pm \frac{d \rho_{k}}{d \phi_{k}} \frac{d \phi_{k}}{d \gamma_{k}}=\frac{d \rho_{k}}{d \gamma_{k}}
$$

In a reference frame not centered at $c_{k}, \mathcal{C}$ has center $c_{k}=r_{k} \mp \rho_{k}$. The time derivative along solutions of (3) gives the velocity of the center

$$
\dot{c}_{k}=s_{k} e^{i \gamma_{k}} \mp \frac{d \rho_{k}}{d \gamma_{k}} \frac{d \gamma_{k}}{d t}=e^{i \gamma_{k}}\left[s_{k} \mp \kappa_{k}^{-1} \nu_{k}\right]
$$

From (30) we see that the curvature control algorithm

$$
\nu_{k}= \pm \kappa_{k} s_{k}
$$

forces $\dot{c}_{k}=0$, implying that the $k^{\text {th }}$ particle drives about a stationary $\mathcal{C}$.

We use Lyapunov analysis to design a decentralized control to collectively steer the particles such that they achieve coincident centers, i.e., $\boldsymbol{c}=\left[\begin{array}{llll}c_{1} & c_{2} & \ldots & c_{N}\end{array}\right]^{T}=c_{0} \mathbf{1}$, where $c_{0} \in \mathbb{C}$ and $\mathbf{1} \in \mathbb{R}^{N}$. Consider the Lyapunov function ${ }^{23}$

$$
S(\mathbf{r}, \gamma)=\frac{1}{2}\langle\mathbf{c}, \mathbf{P c}\rangle
$$

where $\mathbf{P}$ is an $N \times N$ projection matrix, $\mathbf{P}=I_{N \times N}-\frac{1}{N} \mathbf{1 1}^{T} . \mathbf{P}$ defines the communication topology of the system and in this paper corresponds to all-to-all communication. ${ }^{8}$ (Extensions of the flow-free model to limited communication topologies is possible. ${ }^{22}$ ) Equation (32) is positive semi-definite with equality to zero occurring when $\mathbf{c}=c_{0} \mathbf{1}, c_{0} \in \mathbb{C}$. Using 
(30), the time derivative of $(32)$ is

$$
\dot{S}(\mathbf{r}, \boldsymbol{\gamma})=\sum_{k=1}^{N}\left\langle\dot{c}_{k}, P_{k} \mathbf{c}\right\rangle=\sum_{k=1}^{N}\left\langle e^{i \gamma_{k}}, \mathbf{P}_{k} \mathbf{c}\right\rangle\left(s_{k} \mp \kappa_{k}^{-1} \nu_{k}\right)
$$

where $P_{k}$ is the $k^{\text {th }}$ row of $\mathbf{P}$. Notice that the control

$$
\nu_{k}= \pm \kappa_{k}\left(s_{k}+K\left\langle e^{i \gamma_{k}}, \mathbf{P}_{k} \mathbf{c}\right\rangle\right)
$$

substituted into (33) gives

$$
\dot{S}(\mathbf{r}, \boldsymbol{\gamma})=-K \sum_{k=1}^{N}\left\langle e^{i \gamma_{k}}, \mathbf{P}_{k} \mathbf{c}\right\rangle^{2} \leq 0
$$

The invariance principle stipulates that solutions of (5) with control (34) converge to the largest invariant set $\Lambda$ where

$$
\left\langle e^{i \gamma_{k}}, \mathbf{P}_{k} \mathbf{c}\right\rangle \equiv 0
$$

Since $e^{i \gamma_{k}} \neq 0$, then $\mathbf{P}_{k} \mathbf{c}=0$ in $\Lambda$, which is satisfied when the centers are coincident. Moreover, when (36) is satisfied the control in (34) simplifies to (31) which implies $\dot{\boldsymbol{c}}=0$.

A simple example of this control strategy is that of a circular formation. A circle has constant curvature, $\kappa_{k}=\omega_{0}$ for all $k$, which defines a radius of $\left|\omega_{0}\right|^{-1}$. A particle traversing a circular trajectory has inertial velocity tangent to the radius of the circle. Thus, the center of the $k^{\text {th }}$ particle's trajectory with control $\nu_{k}=\omega_{0} s_{k}$ is

$$
c_{k}=r_{k}+\omega_{0}^{-1} \frac{\dot{r_{k}}}{\left|\dot{r}_{k}\right|}=r_{k}+\omega_{0}^{-1} e^{i \gamma_{k}}
$$

The vector $\mathbf{c}$ is then used to calculate the control of $k^{\text {th }}$ particle according to (34) with $\kappa_{k}=\omega_{0}$.

\section{B. Decentralized Multi-vehicle Control in a Strong Flowfield}

In this section we describe the design of a multi-vehicle control law that steers individual particles to a desired feasible formation in a strong flowfield. We establish the stability of multi-vehicle formations in a strong flow when the initial formation centers lie in a feasible region. We use the notation $B(x, a)$ to represent a ball of radius $a$ centered about $x \in \mathbb{C}$.

Theorem 2. Let $\mathcal{F}$ be a feasible region and $c_{0} \in \mathbb{C}$. If $B\left(c_{0},\left|c_{k}(0)-c_{0}\right|\right) \subset \mathcal{F}$, the model (2) with the control algorithm (6) where

$$
\nu_{k}=\operatorname{sat}\left(\omega_{0}\left(s_{k}+K\left\langle e^{i \gamma_{k}}, c_{k}-c_{0}\right\rangle\right) ; \nu_{\max }\right)
$$


and

$$
\nu_{\max }>\max _{k}\left|\omega_{0} s_{k}\right|
$$

forces convergence of the $k^{\text {th }}$ particle to a circular formation of radius $\left|\omega_{0}\right|^{-1}$ centered at $c_{0}$.

Proof. Consider the candidate Lyapunov function

$$
V=\frac{1}{2}\left|c_{k}-c_{0}\right|^{2}
$$

where $c_{k}$ is given by (37). The time derivative of (39) is

$$
\dot{V}=\left\langle c_{k}-c_{0}, \dot{c}_{k}\right\rangle=\left\langle c_{k}-c_{0}, e^{i \gamma_{k}}\right\rangle\left(s_{k}-\omega_{0}^{-1} \nu_{k}\right)
$$

Note from (38) that for $\left|\nu_{k}\right|<\nu_{\max }$

$$
\left\langle c_{k}-c_{0}, e^{i \gamma_{k}}\right\rangle=\frac{\nu_{k}-\omega_{0} s_{k}}{K \omega_{0}}
$$

Substituting this result into (40) gives

$$
\dot{V}=\left(\frac{\nu_{k}-\omega_{0} s_{k}}{K \omega_{0}}\right)\left(s_{k}-\omega_{0}^{-1} \nu_{k}\right)=-\frac{\left(\nu_{k}-\omega_{0} s_{k}\right)^{2}}{K \omega_{0}^{2}} \leq 0 \text {. }
$$

When $\left|\nu_{k}\right|=\nu_{\max }, \dot{V}<0$ since $\nu_{\max }>\max _{k}\left|\omega_{0} s_{k}\right|$. Consequently, $c_{k}$ is bounded within $B\left(c_{0},\left|c_{k}(0)-c_{0}\right|\right) \subset \mathcal{F}$. Moreover, solutions converge to the largest invariant set in which $\dot{V}=0$. From (38) and (40) this set contains solutions for which $\nu_{k}=\omega_{0} s_{k}$, which implies $c_{k}=c_{0}$.

Theorem 2 shows that an individual particle will converge to a reference center provided that the reference center and initial particle center lie within a feasible region. We call this reference center a prescribed center and any particle with information of the prescribed center an informed particle. The following result shows that a multi-vehicle system having initial centers in a feasible region and at least one informed particle will converge to a prescribed center in the same feasible region.

Theorem 3. The model (2) with the control algorithm (6) and

$$
\nu_{k}=\operatorname{sat}\left(\omega_{0}\left[s_{k}+K\left(\left\langle e^{i \gamma_{k}}, \boldsymbol{P}_{k} \boldsymbol{c}\right\rangle+a_{k 0}\left\langle e^{i \gamma_{k}}, c_{k}-c_{0}\right\rangle\right)\right] ; \nu_{\max }\right), \quad K>0,
$$

where $a_{k 0}=1$ if the $k^{\text {th }}$ particle is informed of $c_{0}$ and zero otherwise, forces convergence to 
the circular formation of radius $\left|\omega_{0}\right|^{-1}$ centered at $c_{0}$ if

$$
B\left(c_{0}, \max _{j}\left|c_{j}(0)-c_{0}\right|\right) \subset \mathcal{F},
$$

$a_{k 0} \neq 0$ for at least one particle, and

$$
\nu_{\max }>\max _{k}\left|\omega_{0} s_{k}\right|
$$

Proof. Consider the composite Lyapunov function ${ }^{21}$

$$
S=\frac{1}{2}\langle\mathbf{c}, \mathbf{P c}\rangle+\frac{1}{2} \sum_{k=1}^{N} a_{k 0}\left|c_{k}-c_{0}\right|^{2}
$$

The time derivative of (45) along solutions of (2) is

$$
\dot{S}=\sum_{k=1}^{N}\left(\left\langle e^{i \gamma_{k}}, P_{k} \mathbf{c}\right\rangle+a_{k 0}\left\langle e^{i \gamma_{k}}, c_{k}-c_{0}\right\rangle\right)\left(s_{k}-\omega_{0}^{-1} \nu_{k}\right)
$$

For $\nu_{k}<\nu_{\max }$ notice from (43)

$$
\frac{\nu_{k}-\omega_{0} s_{k}}{K \omega_{0}}=\left\langle e^{i \gamma_{k}}, P_{k} \mathbf{c}\right\rangle+a_{k 0}\left\langle e^{i \gamma_{k}}, c_{k}-c_{0}\right\rangle
$$

giving

$$
\dot{S}=-K \sum_{k=1}^{N} \frac{\left(\nu_{k}-\omega_{0} s_{k}\right)^{2}}{K \omega_{0}^{2}} \leq 0 .
$$

When $\nu_{k}=\nu_{\max }, \dot{S}<0$ since $\nu_{\max }>\max _{k}\left|\omega_{0} s_{k}\right|$. Therefore the collection of particle centers is bounded within the ball (44). Solutions converge to the largest invariant set for which $\dot{S}=0$, which occurs when $\nu_{k}=\omega_{0} s_{k}$ for all $k$, implying from (43)

$$
\left\langle e^{i \gamma_{k}}, \mathbf{P}_{k} \mathbf{c}\right\rangle+a_{k 0}\left\langle e^{i \gamma_{k}}, c_{k}-c_{0}\right\rangle=0
$$

for all $k$.

If $a_{k} 0=0$ for at least one and at most $N-1$ particles, then (47) is satisfied only if $\mathbf{P}_{k} \mathbf{c}=0$, which occurs when $c_{k}=c_{j}$ for all $k$ and $j$. For $k$ with $a_{k 0}=1$, (47) is satisfied only if $c_{k}=c_{0}$. Therefore, $\mathbf{c}$ must satisfy $\mathbf{c}=c_{0} \mathbf{1}{ }^{18}$

Simulation results for an arbitrary flowfield are shown in Figure 5(a) for $\omega_{0}^{-1}=20$, $K=0.1$, and $c_{0}=-50+40 i$. Particle trajectories are shown in blue and the final formation is shown in black. The reference center was chosen to be consistent with the feasibility analysis of Figure 4(a). 


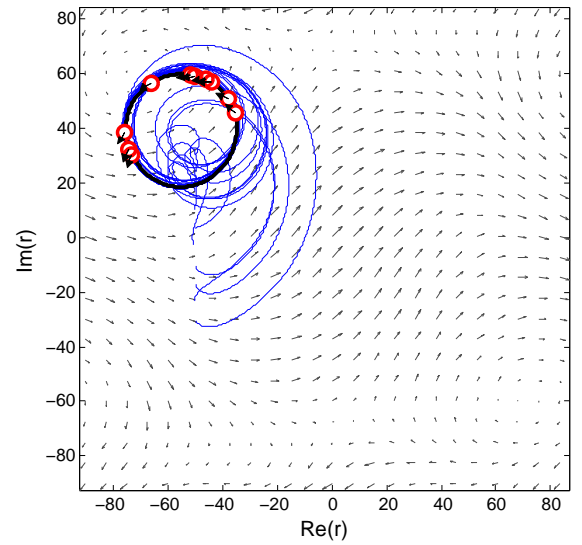

(a)

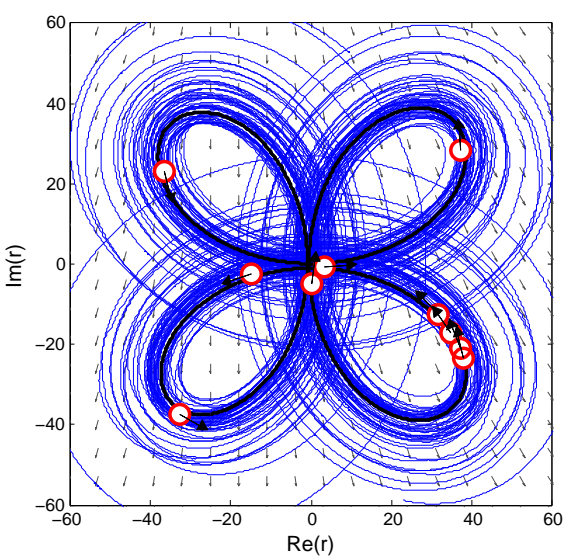

(b)

Figure 5. (a) Simulation of $N=10$ particles with circular control algorithm (43) in a strong flowfield; (b) quadrifolium formation in moderate flowfield.

\section{Stabilization of Folium Formations}

One method for data collection in a hurricane is to fly a figure-four pattern through the center of the hurricane. A flight path with a continuous first derivative covering similar spatial densities is that of the $n$-petalled folium, or polar rose, where $n \geq 3$. Specifically, the quadrifolium $(\mathrm{n}=4)$ is a pattern similar to a figure-four with continuous curvature. In polar coordinates, the equation of the $n$-folium is $\rho=a \sin (g(n) \phi)$, where $\rho$ is the distance from the origin, $\mathrm{n}$ is the number of $\operatorname{lobes}^{\mathrm{c}}, \phi \in[0,2 \pi]$ is the central or polar angle, $a$ is the maximum radius of the lobes, and

$$
g(n)= \begin{cases}n, & n \text { odd } \\ n / 2, & n \text { even }\end{cases}
$$

We showed in Section IV.A that curvature control $\nu_{k}=\kappa_{k} s_{k}$, where $\kappa_{k}=\kappa_{k}\left(\gamma_{k}\right)$ is the curvature, drives the particle along the desired curve. Previous work required the curvature to be nonzero and the figure to be convex..$^{17,23,27}$ Here we relax the convexity assumption. The n-folium is not convex; however, its curvature is strictly positive (or negative). In traversing one rotation about the figure the particle velocity rotates through $2 \pi(g(n)-1)$ radians for $n$ odd and $2 \pi(g(n)+1)$ radians for $n$ even. Using a curvature control $\nu_{k}=\kappa_{k} s_{k}$ requires $\gamma_{k}$ to be defined over $2 \pi(g(n) \pm 1)$ radians. To accomplish this, we augment our state-space equations with a lobe-counter, $l_{k}$, which determines the number of lobes a particle has traversed. As a particle's velocity orientation crosses $2 \pi, l_{k}$ increases by one, giving the

\footnotetext{
${ }^{\mathrm{c}} n$ must be odd or divisible by four to be considered a folium. ${ }^{24}$
} 
following hybrid state-space equations of the $k^{\text {th }}$ particle:

$$
\begin{aligned}
\dot{r}_{k} & =e^{i \theta_{k}}+f_{k} \\
\dot{\theta}_{k} & =u_{k} \\
l_{k}(t+\Delta t) & =l_{k}+\left\lfloor\frac{\gamma_{k}(t)}{2 \pi l_{k}}\right\rfloor,
\end{aligned}
$$

where $\lfloor x\rfloor$ is the largest integer less than $x$ and $l_{k}$ is an integer. Then, by utilizing the curvature control as a function of $\gamma_{k}$ and $l_{k}$ we have

$$
\nu_{k}=\kappa_{k}\left(\gamma_{k}\left(\theta_{k}\right)+2 \pi l_{k}\right) s_{k}
$$

Equation (49) drives the $k^{\text {th }}$ particle about a non-convex figure with strictly positive (negative) curvature.

In the case of the folium, the curvature as a function of the polar angle, $\phi_{k}$, is the polar curvature formula ${ }^{24}$

$$
\kappa_{k}\left(\phi_{k}\right)=\frac{\left|\rho_{k}^{2}+2 \rho_{k}^{\prime 2}-\rho_{k} \rho_{k}^{\prime \prime}\right|}{\left(\rho_{k}^{2}+\rho_{k}^{\prime 2}\right)^{3 / 2}}=\frac{n^{2}+n^{2} \sin ^{2}\left(n \phi_{k}\right)+\cos ^{2}\left(n \phi_{k}\right)}{a\left[\cos ^{2}\left(n \phi_{k}\right)+n^{2} \sin ^{2}\left(n \phi_{k}\right)\right]^{3 / 2}} .
$$

In order to provide state feedback, one must specify the central angle as a function of the relative orientation such that the curvature control law is valid. This relation for the quadrifolium is ${ }^{24}$

$$
\gamma_{k}\left(\phi_{k}\right)=\frac{1}{2} \pi+\phi_{k}-\tan ^{-1}\left(\cot \phi_{k}-\tan \phi_{k}\right)+\pi\left\lfloor 2 \phi_{k} / \pi\right\rfloor
$$

Note the tangential angle is a function of the central angle, not vice versa. To implement (51) we use a look-up table. From (50) and (51) we numerically calculate the curvature as a function of the inertial orientation, such that the control (49) drives the particle around the quadrifolium trajectory. We have the following result, which extends Theorem 3 to curves with definite curvature.

Proposition 1. Consider the quadrifolium with curvature $\kappa_{k}$ given by (50) and $\gamma_{k}$ given by (51). For the model (2), the control algorithm (6) with

$$
\nu_{k}=\kappa_{k}\left[s_{k}+K\left(\left\langle e^{i \gamma_{k}}, \boldsymbol{P}_{k} \boldsymbol{c}\right\rangle+\left\langle e^{i \gamma_{k}}, c_{k}-c_{0}\right\rangle\right)\right]
$$

where $c_{k}=c_{k}\left(\gamma_{k}\right)$ is the instantaneous center of the quadrifolium, drives the $k^{\text {th }}$ vehicle about a quadrifolium formation centered at $c_{0}$.

Simulation results for $N=10$ particles in a quadrifolium formation centered at $c_{0}=0$ 


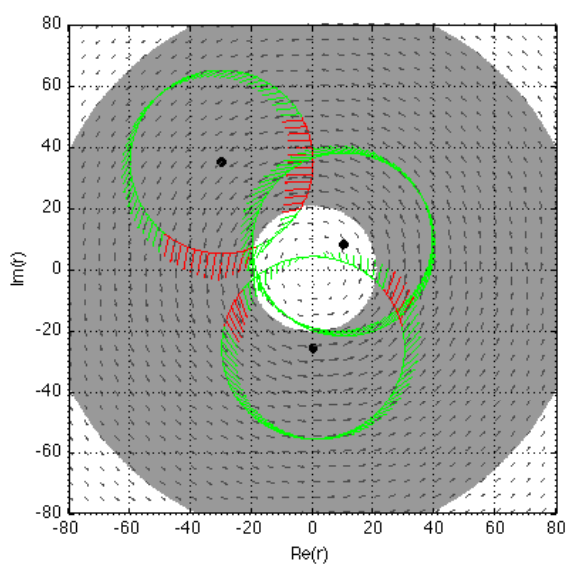

(a)

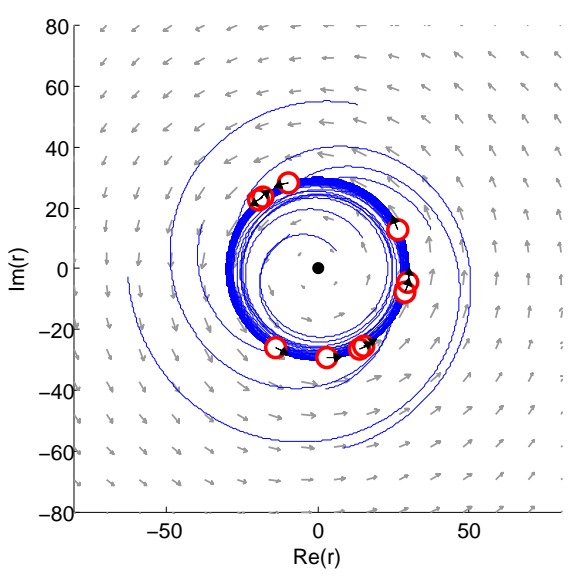

(b)

Figure 6. (a) Regions of achievable centers for counterclockwise circular trajectories of radius $\left|\omega_{0}\right|^{-1}=30$; (b) simulation of the control (43) for $N=10$ particles with $c_{0}=0$.

are shown in Figure 5(b). This simulation utilized a moderate flowfield

$$
f\left(r_{k}\right)=q_{0}\left(\sin \left(2 \pi \omega x_{k}-\psi_{0}\right)-i \cos \left(2 \pi \omega y_{k}-\psi_{0}\right)\right),
$$

with $q_{0}=-0.5, \omega=0.0014$, and $\psi_{0}=3$. Particle trajectories are plotted in blue and the final formation configuration is shown in black.

\section{Design and Stabilization of Hurricane Sampling Patterns}

In this section, we consider a Rankine vortex model of a hurricane. ${ }^{14}$ A Rankine vortex is azimuthally symmetric. The tangential windspeed varies linearly with radius to its maximum $v_{\max }$ at radius $r_{\max }$ and exponentially decreases for radii greater than $r_{\max }$ with exponential decay constant $\alpha$. The Rankine vortex model is ${ }^{14}$

$$
|f(r)|= \begin{cases}v_{\max }\left(|r| / r_{\max }\right), & 0<|r|<r_{\max } \\ v_{\max }\left(|r| / r_{\max }\right)^{-\alpha}, & |r|>r_{\max }\end{cases}
$$

The radius $r=r_{\max }$ represents the eyewall of an idealized hurricane.

Circular formations are an attractive sampling option in a circulating flowfield because large regions of the flowfield are feasible centers of rotation. Figure 6 shows the result of evaluating the kinematic constraint (24) for a circular trajectory of radius $\left|\omega_{0}\right|^{-1}=30$. A drawback of this formation is that there is a fundamental tradeoff between azimuthal and radial sampling densities for a given radius of rotation. For example, a circular formation centered at the origin of a vortex provides good azimuthal sampling but measurements at 


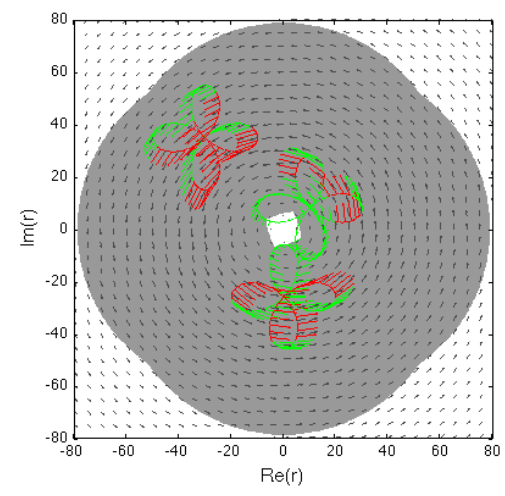

(a)

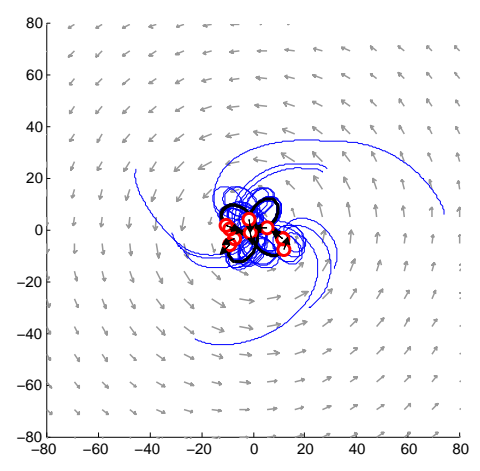

(b)

Figure 7. (a) Regions of achievable centers for quadrifolium trajectories of radius $a=20$; (b) simulation of (43) with $N=10$ particles.

only one radius. A formation not centered at the origin of the vortex provides measurements at multiple radii at the expense of sampling some quadrants of the vortex less frequently than others.

Simulation results of the control algorithm in (43) is shown in Figure 6(b) for Rankine vortex parameters $v_{\max }=1.5, r_{\max }=30$, and $\alpha=0.6$. Notice that the control is calculated based on the inertial variable $\gamma_{k}$, which corresponds to two values of $\theta_{k}$. This implies that the control does not differentiate between aligning or opposing the flowfield.

The same analysis can be used on quadrifolium formations. Figure 7(a) illustrates analysis of the feasibility over a space of centers contained within the Rankine vortex. Figure 7 (b) illustrates simulation of the control (43) with $N=10$ particles. The particle trajectories are shown in blue and the formation after convergence is shown in black. Feasibility analysis reveals that feasible folia are centered close to the origin. With $a=20, r_{\max }=30$, and $v_{\max }=1.5$, quadrifolia centered about the origin are in a moderate flow regime for the majority of the trajectory.

An alternative to circular and folia trajectories that achieve good sampling density and are feasible in a Rankine vortex are spirographs. Spirograph formations are achieved by traversing a circle with respect to a rotating frame. This family of formations combines the advantages of circular and folia formations in that they generally align with the vortex while criss-crossing the eyewall. The feasibility of various spirograph parameter sets in the presence of a Rankine vortex is the subject of ongoing work.

Simulation of the steering control (43) used in the equations of motion (21) for $N=10$ particles is shown in Figure 8. The formation parameters are $\Omega=0.0125 \mathrm{rad} / \mathrm{s}, \omega_{0}^{-1}=40$, and $\rho=30$. A circular formation with respect to a rotating reference frame produces a spirograph formation in an inertial reference frame. Spirograph trajectories provide better radial and azimuthal sampling densities when compared to the circular and quadrifolium 


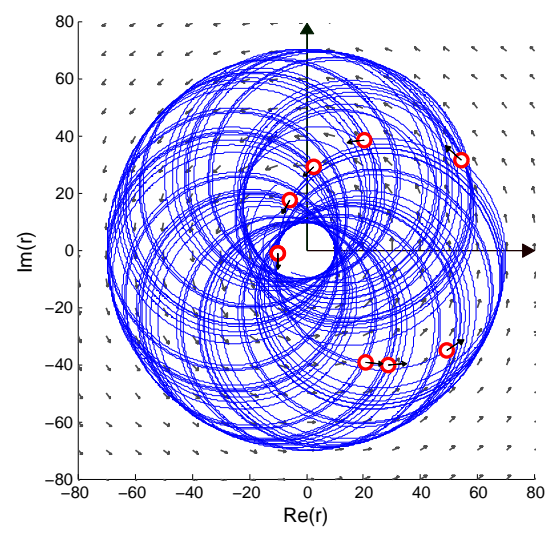

(a)

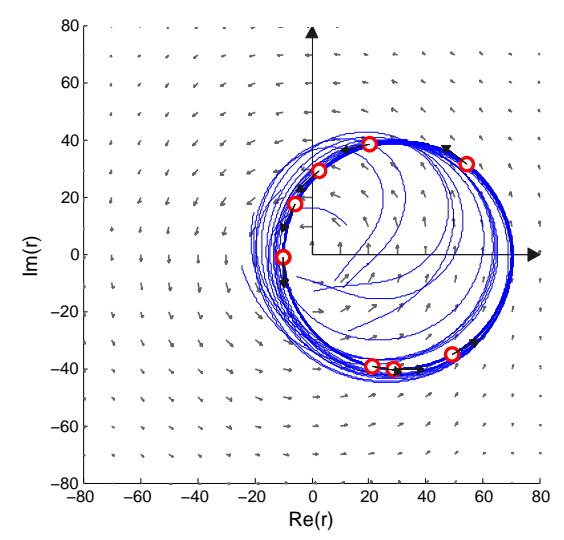

(b)

Figure 8. Spirograph formation produced by utilizing circular control with respect to rotating frame with flow; trajectory in (a) inertial reference frame and (b) rotating reference frame.

formations of Figures 6 and 7.

\section{Conclusion}

A strong flow presents challenges to multi-vehicle autonomous formation control. The presence of strong flows limit the authority of the vehicle to completely dictate its inertial direction of travel, making path planning and collective formation control laws more difficult to design. However, in certain flows, decentralized multi-vehicle control algorithms driving particles to desired formations are feasible. In this paper, we utilize a homogenous vehicle model consisting of unit-speed Newtonian particles with gyroscopic steering control in a flowfield whose flowspeeds can exceed that of the particles. Assuming a known flow, we address the properties a flowfield must satisfy such that given trajectory is feasible. We show that circular formations will converge to a reference center as long as the reference center is feasible. The main results are applied in simulation to an idealized hurricane model. In addition, by using a lobe counting variable, we numerically derive and illustrate a control law driving a collection of particles to a family of non-convex curves called folia, specifically the quadrifolium. These curves closely match that of current hurricane sampling trajectories of manned, and remotely piloted vehicles. Finally, we develop the dynamics of an inertially unit speed particle with respect to a rotating frame to produce spirograph sampling formations. We derive the kinematic feasibility constraints of motion with respect to the rotating frame and simulate vehicle motion in an idealized hurricane model. In ongoing work, we seek to derive control laws converging to a wider set of strong flows, and continue investigation of general flowfield properties which dictate the convergence of multi-vehicle formations. 


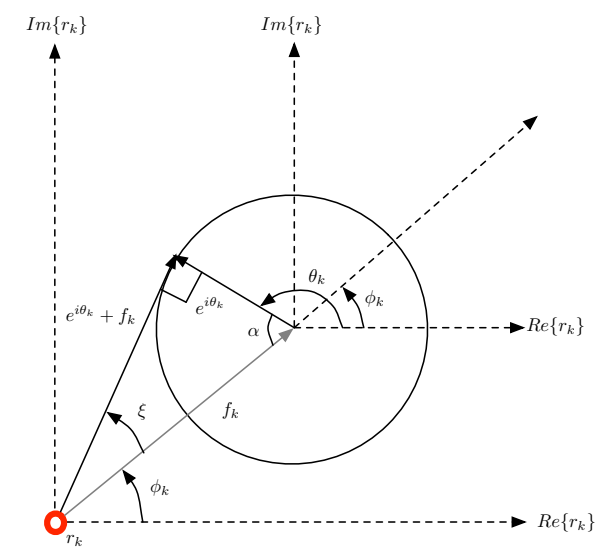

Figure 9. Orientation relations corresponding to singularities of (6).

\section{Appendix}

Proof of Lemma 1: Substituting (3) and (4) into the denominator of (6) implies that $u_{k}$ is singular when

$$
s_{k}=\left\langle e^{i \gamma_{k}}, f_{k}\right\rangle=\left|e^{i \theta_{k}}+f_{k}\right|^{-1}\left\langle e^{i \theta_{k}}+f_{k}, f_{k}\right\rangle=s_{k}^{-1}\left(\left\langle e^{i \theta_{k}}, f_{k}\right\rangle+\left|f_{k}\right|^{2}\right) .
$$

If one considers the flow, $f_{k}$, in its polar form, $f_{k}=\left|f_{k}\right| e^{i \phi_{k}}$, where $\phi_{k}=\arg \left(f_{k}\right)$, substitution into (53) and solving for $\left\langle e^{i \theta_{k}}, e^{i \phi_{k}}\right\rangle$ gives

$$
\left\langle e^{i \theta_{k}}, e^{i \phi_{k}}\right\rangle=\cos \left(\phi_{k}-\theta_{k}\right)=\left|f_{k}\right|^{-1}\left(s_{k}^{2}-\left|f_{k}\right|^{2}\right)=\left|f_{k}\right|^{-1}\left(\left|e^{i \theta_{k}}+f_{k}\right|^{2}-\left|f_{k}\right|^{2}\right)
$$

Notice in Figure 9 that when $e^{i \theta_{k}}$ is drawn such that $\left|e^{i \theta_{k}}+f_{k}\right|$ is tangent to a unit circle drawn about $f_{k}$, a right triangle is formed with hypotenuse $\left|f_{k}\right|$, and sides $\left|e^{i \theta_{k}}+f_{k}\right|$ and $\left|e^{i \theta_{k}}\right|$. Since the vector triad forms a right triangle let $\xi=\sin ^{-1}\left(\left|f_{k}\right|^{-1}\right)$, and since the sum of the interior angles of a triangle are supplementary,

$$
\xi+\alpha+\frac{\pi}{2}=\pi
$$

By projecting $f_{k}$ note also that

$$
\theta_{k}-\phi_{k}+\alpha=\pi
$$

Equating (56) and (55) and taking the cosine of the result implies

$$
\cos \left(\theta_{k}-\phi_{k}\right)=-\left|f_{k}\right|^{-1}
$$

which when substituted into (54) gives the Pythagorean Theorem, $\left|e^{i \theta_{k}}+f_{k}\right|^{2}+1=\left|f_{k}\right|^{2}$. Thus, when $s_{k}=\left\langle e^{i \gamma_{k}}, f_{k}\right\rangle, \theta_{k}$ is such that $e^{i \theta_{k}}, f_{k}$, and $e^{i \theta_{k}}+f_{k}$ form a right triangle. Solving 
(57) for $\theta_{k}$ while noting $\cos \left(\theta_{k}-\phi_{k}\right)=\cos \left(\phi_{k}-\theta_{k}\right)$ completes the proof.

Proof of Lemma 2: The absolute value of (9) squared gives

$$
s_{k}^{2}=\tilde{s}_{k}^{2}+\Omega^{2}\langle\tilde{r}, \tilde{r}\rangle-2 \Omega\left\langle\tilde{r}, i e^{i \tilde{\theta}_{k}}\right\rangle .
$$

Substituting the singular condition $\tilde{s}_{k}=\Omega\left\langle\tilde{r}_{k}, i e^{i \tilde{\theta}_{k}}\right\rangle$ into (58) yields

$$
\begin{aligned}
s_{k}^{2} & =\Omega^{2}\left\langle\tilde{r}_{k}, i e_{k}^{i \tilde{\theta}}\right\rangle^{2}+\Omega^{2}\left\langle\tilde{r}_{k}, \tilde{r}_{k}\right\rangle-2 \Omega^{2}\left\langle\tilde{r}_{k}, i e^{i \tilde{\theta}_{k}}\right\rangle^{2} \\
& =\Omega^{2}\left\langle\tilde{r}_{k}, e^{i \tilde{\theta}_{k}}\right\rangle^{2}
\end{aligned}
$$

revealing a relation between the speed of a particle and its position such that the mapping can become singular

$$
s_{k}=\Omega\left\langle\tilde{r}_{k}, e^{i \tilde{\theta}_{k}}\right\rangle .
$$

Specifically, if the vehicle is assumed to travel at unit speed in the inertial frame the mapping is singular if $\Omega^{-1}=\left\langle\tilde{r}_{k}, e^{i \tilde{\theta}_{k}}\right\rangle$.

\section{Acknowledgments}

The authors would like to acknowledge Angela Maki, Doug Koch, and Sharan Majumdar for discussions contributing to the results of this work. This material is based upon research supported by the National Science Foundation under Grant No. CMMI0928416 and the Office of Naval Research under Grant No. N00014-09-1-1058.

\section{References}

${ }^{1}$ L. Arranz, A. Seuret, and C. Canudas de Wit, Translation control of a fleet circular formation of AUVs under finite communication range, Conference on Decision and Control (Shanghai, China), December 2009, pp. 8345-8350.

2 , Contraction control of a fleet circular formation of AUVs under limited communication range, American Control Conference (Baltimore, MD), June 2010, pp. 5991-5996.

${ }^{3}$ E. Bakolas and P. Tsiotras, Minimum-time paths for a light aircraft in the presence of regionallyvarying strong winds, In AIAA Infotech at Aerospace (Atlanta, GA), AIAA Paper 2010-3380, April 2010.

${ }^{4}$ J. Elston, B. Argrow, E. Frew, and A. Houston, Evaluation of UAS concepts of operation for severe storm penetration using hardware-in-the-loop simulations, AIAA Guidance, Navigation, and Control Conference (Toronto, Canada), August 2010, pp. 8178-8193.

${ }^{5}$ Jack Elston, Semi-autonomous small unmanned aircraft systems for sampling tornadic supercell thunderstorms, Ph.D. thesis, University of Colorado, 2011.

${ }^{6}$ D. R. Thompson et al., Spatiotemporal path planning in strong, dynamic, uncertain currents, IEEE International Conference on Robotics and Automation (Anchorage, AK), 2010, pp. 4778-4783. 
${ }^{7}$ Ruba A. Amarin et al., Estimates of hurricane wind speed measurement accuracy using the airborne hurricane imaging radiometer, Proceedings of the 29th Conference on Hurricane and Tropical Meteorology (Tucson, AZ), May 2010.

${ }^{8}$ J. A. Fax and R. M. Murray, Information flow and cooperative control of vehicle formations, IEEE Transactions on Automatic Control 49 (2004), no. 9, 1465-1476.

${ }^{9}$ E. Frew, D. Lawrence, and S. Morris, Coordinated standoff tracking of moving targets using Lyapunov guidance vector fields, AIAA Journal of Guidance, Control, and Dynamics 31 (2008), 290-306.

${ }^{10}$ W. Ivancic and D. Sullivan, Delivery of unmanned aerial vehicle data, Earth Science Technology Forum (Arlington, VA), June 2010.

${ }^{11}$ E. W. Justh and P. S. Krishnaprasad, Equilibria and steering laws for planar formations, Systems and Control Letters 52 (2003), 25-38.

${ }^{12}$ N. Kasdin and D. A. Paley, Engineering dynamics: A comprehensive introduction, Princeton University Press, 2011.

${ }^{13}$ H. K. Khalil, Nonlinear systems, 3rd ed., Prentice Hall, 2002.

${ }^{14} \mathrm{~A}$. Krener and K. Ide, Measures of unobservability, IEEE Conference on Decision and Control (Shanghai, China), December 2009, pp. 6401-6406.

${ }^{15}$ Lihua Li, G. Heymsfield, J. Carswell, D. Schaubert, M. McLinden, M. Vega, and M. Perrine, Development of the NASA high-altitude imaging wind and rain airborne profiler, 2011 IEEE Aerospace Conference, March 2011, pp. 1-8.

${ }^{16} \mathrm{P}$. Lin, Observations: The first successful typhoon eyewall-penetration reconnaissance flight mission conducted by the unmanned aerial vehicle, Aerosonde, Bulletin of the American Meteorological Society $\mathbf{8 7}$ (2006), 1481-1483.

${ }^{17}$ D. A. Paley, N. Leonard, and R. Sepulchre, Stabilization of symmetric formations to motion around convex loops, Systems and Control Letters 57 (2006), 209-215.

${ }^{18}$ D. A. Paley and C. Peterson, Stabilization of collective motion in a time-invariant flowfield, AIAA Journal of Guidance, Control, and Dynamics 32 (2009), 771-779.

${ }^{19}$ C. Peterson and D. A. Paley, Multi-vehicle coordination in an estimated time-varying flowfield, AIAA Journal of Guidance, Control, and Dynamics 34 (2011), 177-191.

${ }^{20} \mathrm{R}$. Rysdyk, Course and heading changes in significant wind, AIAA Journal of Guidance, Control, and Dynamics 30 (2007), 1168-1171.

${ }^{21}$ R. Sepulchre, D. A. Paley, and N. E. Leonard, Stabilization of planar collective motion: All-to-all communication, IEEE Transactions on Automatic Control 52 (2007), 811-824.

22 , Stabilization of planar collective motion with limited communication, IEEE Transactions on Automatic Control 53 (2008), 706-719.

${ }^{23}$ L. Techy, D. A. Paley, and C.A. Woolsey, UAV coordination on closed convex paths in wind, AIAA Journal of Guidance, Control, and Dynamics 33 (2010), 1946-1951.

${ }^{24}$ Eric C. Weisstein, "quadrifolium", http://mathworld.wolfram.com/Quadrifolium.html, January 2011.

${ }^{25}$ L.L. Whitcomb, M.V. Jakuba, J.C. Kinsey, S.C. Martin, S.E. Webster, J.C. Howland, C.L. Taylor, D. Gomez-Ibanez, and D.R. Yoerger, Navigation and control of the Nereus hybrid underwater vehicle for global ocean science to 10,903 $\mathrm{m}$ depth: Preliminary results, Robotics and Automation (ICRA), 2010 IEEE International Conference on, May 2010, pp. 594-600.

${ }^{26} \mathrm{H}$. Willoughby and S. Majumdar, personal communication, July 2011. 
${ }^{27}$ F. Zhang and N. E. Leonard, Coordinated patterns of unit speed particles on a closed curve, Systems and Control Letters 56 (2007), 397-407. 